\title{
Lymphoproliferative disorder in an elderly rheumatoid arthritis patient after longterm oral methotrexate administration: A case report
}

\author{
KAZUHIKO HASHIMOTO and MASAO AKAGI \\ Department of Orthopedic Surgery, Kindai University Hospital, Osaka-Sayama, Osaka 589-8511, Japan
}

Received June 21, 2018; Accepted July 18, 2018

DOI: $10.3892 / \mathrm{mco} .2018 .1675$

\begin{abstract}
Methotrexate-associated lymphoproliferative disorder (MTX-LPD) is frequently reported in the literature; however, its pathophysiology has not been fully elucidated to date. We herein describe a case of MTX-LPD that occurred after long-term treatment with oral MTX in a 67-year-old Japanese woman with rheumatoid arthritis (RA) who presented with generalized lymphadenopathy of the neck. The patient had been diagnosed with RA 24 years earlier, and had been on oral MTX for 20 years. The patient noticed a mass on her neck, which prompted a visit to our hospital. The mass was confirmed as diffuse large B-cell lymphoma by biopsy. MTX treatment was discontinued, which resulted in a reduction in the size of the mass and improvement of the patient's symptoms. Therefore, clinicians must be aware of MTX-LPD as a differential diagnosis for patients with rheumatological conditions on long-term MTX therapy presenting with signs and symptoms suggestive of lymphoma.
\end{abstract}

\section{Introduction}

The treatment of rheumatoid arthritis (RA) has evolved in recent years. Methotrexate (MTX), with or without biologics, is currently the first-line therapy for RA, and is widely administered as an anchor drug (1). The incidence of lymphoproliferative disorder (LPD) is 2.0-5.5 times higher in patients with RA compared with that in the general population, and MTX has been described as a major cause of LPD (2). The World Health Organization classification of lymphoid neoplasms describes MTX-related LPD as an 'iatrogenic immunodeficiency-associated LPD', which is similar to immunodeficiency-associated LPD, including post-transplant LPD and human immunodeficiency-associated LPD (3).

Correspondence to: Dr Kazuhiko Hashimoto, Department of Orthopedic Surgery, Kindai University Hospital, 3772 OhnoHigashi, Osaka-Sayama, Osaka 589-8511, Japan

E-mail: hazzhiko@med.kindai.ac.jp

Key words: methotrexate-associated lymphoproliferative disorder, elderly, rheumatoid arthritis, long-term oral administration
Approximately 40-50\% of MTX-related LPD (MTX-LPD) cases occur in extranodal sites, with the gastrointestinal tract, skin, liver, lung and kidney reported as the most susceptible (4,5). The onset of MTX-LPD is shorter compared with that of lymphomas that develop in general RA patients (6). The causative role of MTX in MTX-LPD pathophysiology is supported by the observation that discontinuing treatment with MTX results in resolution of LPD (7). The onset of MTX-LPD is considered to be dose-independent, as is the case with MTX-related interstitial pneumonia (8). Previous studies to date have demonstrated that the mean duration and mean total dose of MTX were 38-54 months and 940-984 mg, respectively $(6,7)$. We herein describe a case of MTX-LPD with the longest duration of MTX treatment identified in the literature to date, and the highest total dose of MTX in a patient with RA.

\section{Case report}

A 67-year-old woman presented to the Kindai University Hospital (Osaka, Japan) in May 2012 with complaints of fever and neck swelling. The patient had a history of RA, for which she had been receiving oral methotrexate (MTX) $8 \mathrm{mg} /$ week, prednisolone $2.5 \mathrm{mg} /$ day and bucillamine (BUC) $100 \mathrm{mg} /$ day. The patient had been taking MTX for 20 years, and her cumulative dose at the time of presentation to our hospital was 7,636 mg. Radiography revealed destruction of cartilage and bone, and joint hyperextension (Fig. 1A). The patient was diagnosed with stage III disease according to the Steinbrocker classification (9). There was no evidence of lung involvement on radiographic examination (data not shown). Clinical examination of the neck revealed several non-tender, elastic, fluctuant, solid masses, measuring $\sim 1-3 \mathrm{~cm}$ in greatest diameter. The masses were well-adhered to the overlying skin. Blood test results revealed an inflammatory response, high soluble IL-2 receptor (sIL-2R) levels, and EbsteinBarr virus (EBV) antibody positivity (Table I). Computed tomography revealed an isointense mass in the right side of the neck (Fig. 1B), which was also noted on T1-weighted magnetic resonance imaging (MRI) (Fig. 1C). T2-weighted MRI revealed a high-intensity mass (data not shown). Positron emission tomography with 2-deoxy-2-(fluorine-18)-fluoroD-glucose integrated with computed tomography $\left({ }^{18} \mathrm{~F}-\mathrm{FDG}\right.$ $\mathrm{PET} / \mathrm{CT}$ ) demonstrated FDG accumulation in the right neck mass (Fig. 1D). Based on these findings, needle biopsy of 
the mass was performed. Histological examination of the bioptic material revealed increased proliferation of atypical lymphocytes with nuclear enlargement, and lymph node structure with ambiguous histology on hematoxylin and eosin staining (Fig. 2A and B). Following discontinuation of oral MTX and BUC treatment, the lymphadenopathy temporarily disappeared. However, the right cervical lymphadenopathy became more prominent $\sim 1$ month later. Repeat laboratory investigations at that time revealed elevated levels of sIL-2R and lactate dehydrogenase, which was consistent with disease relapse. Therefore, the patient was initiated on high-dose steroid therapy. Complete remission was observed at 4 years after treatment, and there was no recurrence at 6 months after the end of treatment. Treatment for RA was resumed, and good control was obtained with prednisolone $2.5 \mathrm{mg} /$ day and salazosulfapyridine $1,000 \mathrm{mg} / \mathrm{day}$. The patient has subsequently been followed up every 5 years.

\section{Discussion}

MTX-LPD is a serious complication in patients treated with MTX. The incidence of MTX-LPD has increased, due in part to the increased use of MTX as an anchor drug in the management of RA. Given the important implications, there is a need to further elucidate the incidence, demographic characteristics and risk factors for this condition. To this end, we herein describe a case of MTX-LPD in an elderly RA patient on longterm oral treatment with MTX.

Although the mechanism of onset is unknown, the combination of immunodeficiency as a result of RA and the immunosuppressive effect of MTX has been implicated in the pathogenesis of MTX-LPD $(5,6)$. In RA, self-reactive T cells of a specific clone stimulate $\mathrm{B}$ cells that produce autoantibodies, such as rheumatoid factor (10). When MTX is administered, its immunosuppressive effect reactivates viral infections, including subclinical infections, and it is hypothesized that this triggers clonal proliferation of cells (11). In particular, EBV is considered to be an important viral infection associated with LPD that develops in the context of rheumatological diseases (8). Activation of EBV is identified in the majority of MTX-LPD cases (8), including the present case, which was characterized by the presence of positive EBV antibody serology.

The clinical characteristics of MTX-LPD include superficial and deep lymphadenopathy, as well as systemic symptoms, such as fever and weight loss $(5,6)$. The frequency of extralymphatic involvement is relatively high, with lesions most commonly affecting the skin, soft tissues and lung $(5,6,11)$. In the present case, no lung lesions were observed.

Although the histological characteristics of MTX-LPD are diverse, the most common finding is diffuse large B-cell lymphoma (DLBCL), which accounts for $\sim 50 \%$ of all cases, followed by Hodgkin's lymphoma (HL) in $20 \%$ of the cases $(5,12)$. The patient in the present case was diagnosed with DLBCL. A characteristic feature of MTX-LPD pathology is the presence of $\mathrm{B}, \mathrm{T}$ and natural killer cells, as well as other cell subtypes derived from lymphocytes (12). Complicated phenotypes, including pathological findings of DLCBCL mixed with lymphoma-like granulomatosis and peripheral T-cell lymphoma, non-specific type, have also been reported (12).
Table I. Blood test results.

\begin{tabular}{lc}
\hline Tests (units) & Values \\
\hline C-reactive protein $(\mathrm{mg} / \mathrm{dl})$ & 1.41 \\
Aspartate transaminase $(\mathrm{U} / \mathrm{l})$ & 36 \\
Alanine transaminase $(\mathrm{U} / \mathrm{l})$ & 25 \\
White blood cells $\left(\mathrm{x} 10^{3} \mu \mathrm{l}\right)$ & 7.3 \\
Hemoglobin $(\mathrm{g} / \mathrm{dl})$ & 13.7 \\
Platelets $\left(\mathrm{x} 10^{4} \mu \mathrm{l}\right)$ & 18.8 \\
Blood urea nitrogen $(\mathrm{mg} / \mathrm{dl})$ & 16 \\
Creatinine $(\mathrm{mg} / \mathrm{dl})$ & 0.64 \\
IgG & 1,003 \\
sIL-2R (U/ml) & 2,067 \\
$\beta 2-M G(\mathrm{mg} / \mathrm{dl})$ & 3.2 \\
EBV VCA IgG & $7.9(+)$ \\
Squamous cell carcinoma tumor marker & $(-)$ \\
\hline
\end{tabular}

EBV, Epstein-Barr virus; IgG, immunoglobulin G; sIL-2R, soluble interleukin-2 receptor; $\mathrm{MG}$, microglobulin; VCA, viral capsid antigen.

A previous report suggested that other immunosuppressants also contribute to the onset of MTX-LPD (13). However, no study to date has implicated BUC. The patient described herein was receiving both MTX and BUC; therefore, the possible involvement of BUC in the development of MTX-LPD cannot be excluded.

The duration and dose of MTX treatment has varied across studies. A previous report of an RA patient receiving MTX therapy who developed MTX-LPD described a median duration of 38 months and total dose of $984 \mathrm{mg}$ (7). Another previous study reported a median duration and total dose of 54 months and $940 \mathrm{mg}$, respectively (6). To the best of our knowledge, the patient described in the present case had the most delayed onset of MTX-LPD following initiation of MTX treatment, and the highest cumulative dose of MTX prior to disease onset.

Spontaneous resolution of LPD after withdrawal of MTX treatment is observed in $~ 50 \%$ of all affected patients $(12,14,15)$. However, cases of patients with LPD progression after the discontinuation of MTX have also been reported $(12,15)$. The progression or resolution of LPD is not only affected by MTX treatment per se, but also by the immune status of the host, which, as previously described, is affected by RA pathogenesis. However, the majority of reports recommend interrupting MTX treatment upon development of MTX-LPD (12,14-16). In a previous study, 23 patients $(76.7 \%)$ achieved regression of LPD by MTX withdrawal (16). In another study, the majority of DLBCLtype MTX-LPD patients (81\%) achieved remission with MTX discontinuation alone (17). By contrast, the majority of patients with classical HL-type MTX-LPD (76\%) required additional chemotherapy (17). Corticosteroids are used in combination with cyclophosphamide or chemotherapy for the treatment of malignant lymphoma, whereas a previous study reported the use of rituximab (18). In keeping with our results, another study also demonstrated that corticosteroid 

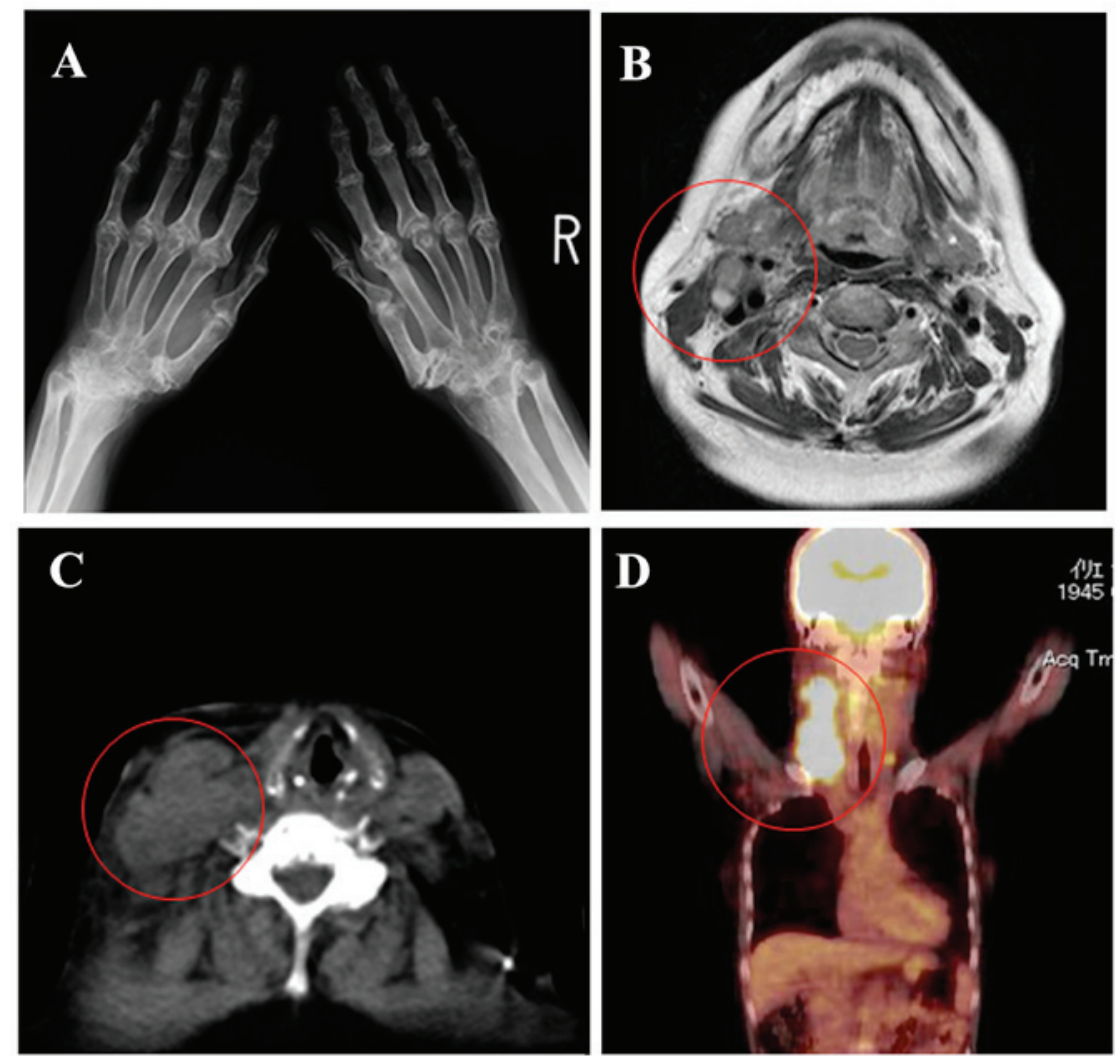

Figure 1. (A) Radiography of both hands showing joint space narrowing, with bone and joint hyperextension. (B) Computed tomography of the patient's neck showing an isointense mass (red circle) in the right neck. (C) T1-weighted magnetic resonance image (MRI) of the patient's neck showing an isointense mass in the right neck. (D) ${ }^{18} \mathrm{~F}$-fluorodeoxyglucose positron emission tomography integrated with computed tomography of the patient's neck revealed FDG accumulation in the right neck mass.
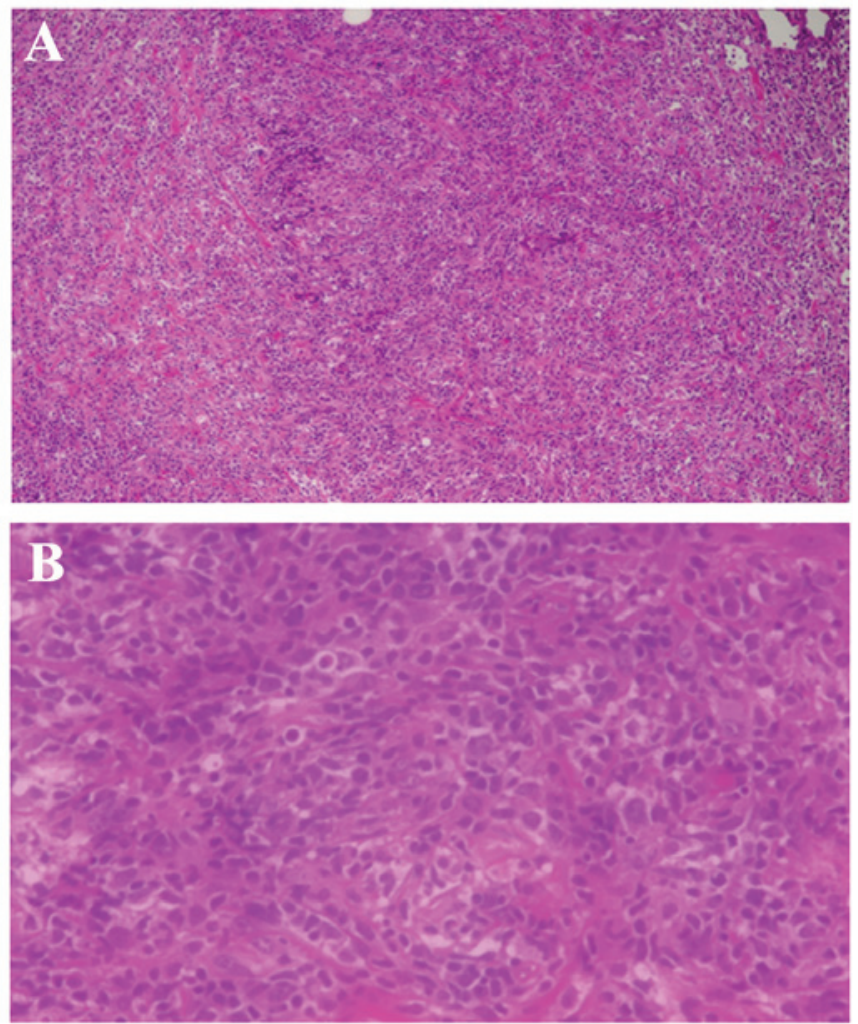

Figure 2. Examination of hematoxylin and eosin-stained sections of the mass revealed increased proliferation of atypical lymphocytes with nuclear enlargement and ambiguous lymph node structure. Scale bar, $100 \mu \mathrm{m}$. Magnification, (A) x40 and (B) x400. pulse therapy was effective (12). EBV has been described as an important prognostic factor. A previous study reported that a significantly higher positive rate of peripheral blood EBV DNA was observed in MTX-LPD patients belonging to the chemotherapy-free group compared with patients requiring chemotherapy ( $9 / 9$ vs. $0 / 3$, respectively; $\mathrm{P}=0.0002)$, suggesting that EBV DNA positivity in the peripheral blood is a marker of better outcome in these patients (16). This finding is also consistent with our experience, as the patient in the present case was EBV-positive and developed no recurrence.

In conclusion, we herein reported a case of LPD in an elderly female RA patient with a history of long-term oral MTX administration. In order to facilitate early diagnosis and intervention, clinicians must be aware of the risk of MTX-LPD in such patients.

\section{Acknowledgements}

Not applicable.

\section{Funding}

No funding was received.

\section{Availability of data and materials}

Not applicable. 


\section{Authors' contributions}

Data was acquired and analyzed by KH and MA. KH and MA prepared the manuscript.

\section{Ethics approval and consent to participate}

The patient gave their consent to participate in this study.

\section{Patient consent for publication}

Consent for publication of the case details and associated images was obtained from the patient.

\section{Competing interests}

All the authors declare that they have no competing interests to disclose.

\section{References}

1. Smolen JS,LandewéR,Bijlsma J,Burmester G, ChatzidionysiouK, Dougados M, Nam J, Ramiro S, Voshaar M, van Vollenhoven R, et al: EULAR recommendations for the management of rheumatoid arthritis with synthetic and biological disease-modifying antirheumatic drugs: 2016 update. Ann Rheum Dis 76: 960-977, 2017.

2. Baecklund E, Iliadou A, Askling J, Ekbom A, Backlin C, Granath F, Catrina AI, Rosenquist R, Feltelius N, Sundström C, et al: Association of chronic inflammation, not its treatment, with increased lymphoma risk in rheumatoid arthritis. Arthritis Rheum 54: 692-701, 2006.

3. Ellman MH, Hurwitz H, Thomas C and Kozloff M: Lymphoma developing in a patient with rheumatoid arthritis taking low dose weekly methotrexate. J Rheumatol 18: 1741-1743, 1991.

4. Ishiguro K, Hayashi T, Aoki Y, Murakami R, Ikeda H and Ishida T: Other iatrogenic immunodeficiency-associated lymphoproliferative disorder presenting as primary bone lymphoma in a patient with rheumatoid arthritis. Intern Med 55: 2259-2264, 2016.

5. Kameda T, Dobashi H, Miyatake N, Inoo M, Onishi I, Kurata N, Mitsunaka H, Kawakami K, Fukumoto T, Susaki K, et al: Association of higher methotrexate dose with lymphoproliferative disease onset in rheumatoid arthritis patients. Arthritis Care Res (Hoboken) 66: 1302-1309, 2014.

6. Hoshida Y, Xu JX, Fujita S, Nakamichi I, Ikeda J, Tomita Y, Nakatsuka S, Tamaru J, Iizuka A, Takeuchi $\mathrm{T}$, et al: Lymphoproliferative disorders in rheumatoid arthritis: Clinicopathological analysis of 76 cases in relation to methotrexate medication. J Rheumatol 34: 322-331, 2007.

7. Miyazaki T, Fujimaki K, Shirasugi Y, Yoshiba F, Ohsaka M, Miyazaki K, Yamazaki E, Sakai R, Tamaru J, Kishi K, et al: Remission of lymphoma after withdrawal of methotrexate in rheumatoid arthritis: Relationship with type of latent Epstein-Barr virus infection. Am J Hematol 82: 1106-1109, 2007.
8. Mariette X, Cazals-Hatem D, Warszawki J, Liote F, Balandraud N and Sibilia J; Investigators of the Club Rhumatismes et Inflammation: Lymphomas in rheumatoid arthritis patients treated with methotrexate: A 3-year prospective study in France. Blood 99: 3909-3915, 2002.

9. Matsuda K, Gotoh M, Mitsui Y, Yoshikawa E, Kume S, Yano M, Honda S, Okawa T, Fukuda T, Higuchi F, et al: Steinbrocker classification class IV to class II after multi-joint surgery. Kurume Med J. 59:7 9-82, 2012.10.

10. Lu DR, McDavid AN, Kongpachith S, Lingampalli N, Glanville J, Ju CH, Gottardo R, Robinson WH: T cell-dependent affinity maturation and innate immune pathways differentially drive autoreactive B cell responses in rheumatoid arthritis. Arthritis Rheumatol: May 31,2018 (Epub ahead of print).

11. Mokuda S, Miyazaki T, Saeki Y, Masumoto J, Kanno M and Takasugi K: Epstein-Barr virus-related MTX-LPD in rheumatoid arthritis patients exhibits a viral pattern of the CD64 and CD35 expression on neutrophils: Three case reports. Mod Rheumatol 25: 166-168, 2015.

12. Tokuhira M, Watanabe R, Nemoto T, Sagawa M, Tomikawa T, Tamaru J, Itoyama S, Nagasawa H, Amano K, Kameda H, et al: Clinicopathological analyses in patients with other iatrogenic immunodeficiency-associated lymphoproliferative diseases and rheumatoid arthritis. Leuk Lymphoma 53: 616-623, 2012.

13. Hashimoto A, Chiba N, Tsuno H, Komiya A, Furukawa H, Matsui T, Nishino $J$ and Tohma S: Incidence of malignancy and the risk of lymphoma in Japanese patients with rheumatoid arthritis compared to the general population. J Rheumatol 42: 564-571, 2015.

14. Ureshino H, Kadota C, Kurogi K, Miyahara M and Kimura S: Spontaneous Regression of Methotrexate-related Lymphoproliferative Disorder with T-cell Large Granular Lymphocytosis. Intern Med 54: 2235-2239, 2015.

15. Ichikawa A, Arakawa F, Kiyasu J, Sato K, Miyoshi H, Niino D, Kimura Y, Takeuchi M, Yoshida M, Ishibashi Y, et al: Methotrexate/iatrogenic lymphoproliferative disorders in rheumatoid arthritis: Histology, Epstein-Barr virus, and clonality are important predictors of disease progression and regression. Eur J Haematol 91: 20-28, 2013.

16. Katsuyama T, Sada KE, Yan M, Zeggar S, Hiramatsu S, Miyawaki Y, Ohashi K, Morishita M, Watanabe H, Katsuyama E, et al: Prognostic factors of methotrexate-associated lymphoproliferative disorders associated with rheumatoid arthritis and plausible application of biological agents. Mod Rheumatol 27: 773-777, 2017.

17. Zaidi A, Kampalath B, Peltier WL and Vesole DH: Successful treatment of systemic and central nervous system lymphomatoid granulomatosis with rituximab. Leuk Lymphoma 45: 777-780, 2004.

18. Gion Y, Iwaki N, Takata K, Takeuchi M, Nishida K, Orita Y, Tachibana T, Yoshino T and Sato Y: Clinicopathological analysis of methotrexate-associated lymphoproliferative disorders: Comparison of diffuse large B-cell lymphoma and classical Hodgkin lymphoma types. Cancer Sci 108: 1271-1280, 2017. 